\title{
GEOMETRY OF INFINITELY PRESENTED SMALL CANCELLATION GROUPS AND QUASI-HOMOMORPHISMS
}

\author{
GOULNARA ARZHANTSEVA AND CORNELIA DRUŢU
}

\begin{abstract}
We study the geometry of infinitely presented groups satisfying the small cancellation condition $C^{\prime}(1 / 8)$, and introduce a standard decomposition (called the criss-cross decomposition) for the elements of such groups. Our method yields a direct construction of a linearly independent set of power continuum in the kernel of the comparison map between the bounded and the usual group cohomology in degree 2, without the use of free subgroups and extensions.
\end{abstract}

\section{INTRODUCTION}

An important direction of research in geometric group theory is the construction of infinite finitely generated groups with unusual properties, the so-called "infinite monsters". In this setting, one of the main techniques is small cancellation theory. It produces direct limits of Gromov hyperbolic groups that are therefore, in the class of infinitely presented groups, easier to deal with than other groups, and benefit in many ways from the techniques available in Gromov hyperbolic geometry. These, and other, infinite monsters are usually constructed with the goal of producing counter-examples to various conjectures in algebra, geometry, and analysis. Therefore, it is rather challenging to obtain positive results about them. Positive results have been proved for algebraic and geometric properties, and much less for analytic properties, until very recently. The present paper and its successor [AD17] have been first steps in this direction. The first arXiv version of this paper has already given an impetus to later works by various authors.

One of the first and easiest constructions of small cancellation groups is that of groups satisfying the classical small cancellation condition $C^{\prime}(\lambda)$, where $\lambda \in(0,1 / 6]$. It seems likely that most of the properties of Gromov hyperbolic groups are also satisfied by such groups, possibly for $\lambda$ small enough, but this is not yet proved for many of the analytic properties of hyperbolic groups.

In this paper, we provide a key tool for a systematic approach to the above mentioned problem, the criss-cross decomposition. As an immediate application, we provide a new way of constructing an abundance of quasi-homomorphisms in these groups, see Section 1.2 and Theorem 4.6. Moreover, our quasi-morphisms are

2010 Mathematics Subject Classification. 20F06, 20F67.

Key words and phrases. Small cancellation theory, Greendlinger lemma, quasihomomorphisms, bounded cohomology.

The first author was supported in part by the ERC grant ANALYTIC no. 259527, and by the Swiss NSF, under Sinergia grant CRSI22-130435.

The second author was supported in part by the EPSRC grant no. EP/K032208/1 entitled "Geometric and analytic aspects of infinite groups", by the project ANR Blanc ANR-10-BLAN 0116, acronym GGAA, and by the LABEX CEMPI. 
$\mathbb{Z}$-valued and have defect at most 2. This may be significant because such a bound on the defect has consequences for the stable commutator length [Bav91].

Our methods very likely generalize to larger classes of groups.

1.1. Main technical tool: criss-cross decompositions. For several analytic and geometric properties of a group, including the one discussed in this paper, it is crucial to understand if the group elements possess "standard decompositions" into products of certain "elementary" parts.

The main technical result of our paper is the construction of such a decomposition for elements of finitely generated groups defined by infinite presentations with the small cancellation condition $C^{\prime}(\lambda)$, for $\lambda \leqslant \frac{1}{8}$ (the so-called $C^{\prime}(1 / 8)$-groups). More precisely, given a pair of vertices in a Cayley graph of such a group, we obtain a detailed description of a set containing all the geodesics between the two vertices, see Theorem 3.15. The existence of such sets allows us to introduce a uniquely defined decomposition, called the criss-cross decomposition, of the elements of the given $C^{\prime}(1 / 8)$-group, see Section 3.

It is worth noticing that our approach differs and cannot be deduced from the Rips-Sela canonical representatives [Sel92, RS95] in finitely presented small cancellation groups. Indeed, the Rips-Sela construction gives an equivariant choice of quasi-geodesic paths between pairs of vertices (with a view to reduce solving equations in a finitely presented small cancellation group, or more generally in a hyperbolic group, to solving equations in a free group). Their arguments are based on the existence of central points for geodesic triangles, granted by finite presentation only. The similarity in method between the Rips-Sela approach and ours does not go beyond the common use of the geometry of geodesic bigons [RS95, Theorem 5.1].

1.2. Application: the bounded versus the usual cohomology. An immediate application of the criss-cross decomposition is that infinitely presented $C^{\prime}(1 / 12)-$ groups are rich in quasi-homomorphisms (see Section 4 for definitions). This has a strong impact on the bounded cohomology of such groups [Gro82]. We thus deduce the following theorem.

Theorem 1.1. Let $G$ be a finitely generated group defined by an infinite presentation satisfying the small cancellation condition $C^{\prime}(1 / 12)$. Then the kernel of the comparison map between the second bounded and the usual group cohomology

$$
H_{b}^{2}(G) \rightarrow H^{2}(G),
$$

is an infinite dimensional real vector space, with a basis of power continuum.

The above kernel can be identified with the real vector space $\widetilde{Q H}(G)$ of quasihomomorphisms modulo near-homomorphisms (where by a near-homomorphism we mean a function $\mathfrak{h}: G \rightarrow \mathbb{R}$ that differs from a homomorphism by a bounded function). The fact that the kernel is large has implications for the stable commutator length [Bav91], for the (lack of) bounded generation, for results of nonembeddability of higher rank lattices, etc.

The computation of $\widetilde{Q H}(G)$ is therefore important, and it has been done for various classes of groups.

If $G$ is amenable then $\widetilde{Q H}(G)=0$ [Gro82]. Also, if $G$ is an irreducible lattice in a semisimple Lie group of rank at least two and with finite center then $\widetilde{Q H}(G)=0$ [BM99, BM02]. 
Groups that have a certain type of action on a hyperbolic space (in particular, subgroups of relatively hyperbolic groups, mapping class groups, etc.) have $\widetilde{Q H}(G)$ infinite dimensional, with a basis of power continuum. This was proved by Brooks for non-abelian free groups [Bro81] and by Brooks and Series for non-amenable surface groups. In [Gro87] Gromov stated that all non-elementary hyperbolic groups have non-trivial second bounded cohomology. Epstein and Fujiwara proved that, in fact, for all non-elementary hyperbolic groups $\widetilde{Q H}(G)$ has a basis of power continuum [EF97]. Later this result was extended to other types of groups acting on hyperbolic spaces and to their non-elementary subgroups [Fuj00, Fuj98]; in particular, to subgroups of mapping class groups of surfaces [BF02]. See also the survey of Fujiwara [Fuj09] and references therein. The same result was further extended to groups with free hyperbolically embedded subgroups by Hull and Osin [HO13].

On the whole one can say that in all the cases where it was proved, up to now, that $\widetilde{Q H}(G)$ has a basis of power continuum, the argument relied on the fact that the group considered contained a non-elementary hyperbolic subgroup, and an extension of the quasi-morphisms of that subgroup could be perfomed, if the subgroup was "well embedded" (e.g. hyperbolically embedded, in the sense of [DGO17]). In particular, two years after the first arXiv version of this paper was posted, it has been proven by Gruber and Sisto in [GS14] that graphical small cancellation groups are acylindrically hyperbolic, therefore, by work of Hull and Osin [HO13], contain hyperbolically embedded free non-abelian subgroups, and consequently the space of quasi-homomorphisms modulo near-homomorphisms has a basis of power continuum.

Our approach differs from all the previous ones in that we do not require the existence of "well embedded" non-elementary hyperbolic subgroups, and a potential extension of our methods may apply to groups satisfying other small cancellation conditions (e.g. the Ol'shanskii graded small cancellation), in particular, to free Burnside groups of sufficiently large odd exponent or to various Tarski monsters.

Moreover, besides being by far the first proof of Theorem 1.1, our construction has the merit of by-passing the technicalities of the two papers [GS14] and [HO13] and of providing a direct explicit construction of a family of power continuum of linearly independent elements in $\widetilde{Q H}(G)$, which is not an extension of a similar family for a non-elementary hyperbolic subgroup $H \leqslant G$, but is contained in the $\ell^{1}$ infinite sum of all such extensions for all such subgroups $H$.

The following result is another immediate consequence of our theorem above.

Corollary 1.2. Let $G$ be a finitely generated group given by an infinite presentation satisfying the small cancellation condition $C^{\prime}(1 / 12)$. Then $G$ is not boundedly generated ${ }^{1}$.

1.3. Plan of the paper. The paper is organized as follows. Section 2 gives preliminary information on small cancellation groups. In Section 3, we describe the crisscross decomposition of elements in infinitely presented small cancellation groups. We believe this description is of independent interest and it can be applied to get further results on such groups. In Section 4 we focus on quasi-homomorphisms of $C^{\prime}(1 / 12)$-small cancellation groups and prove Theorem 1.1.

\footnotetext{
${ }^{1} \mathrm{~A}$ group is boundedly generated if it can be expressed (as a set) as a finite product of cyclic subgroups.
}

Copyright (c) 2018 Canadian Mathematical Society. All rights reserved. 
Acknowledgments. The work on this paper was carried out during visits of the first author to the University of Oxford and of the second author to the University of Vienna. We thank these institutions for their support and hospitality. The first author is also grateful to the ETH Zürich and to the CRM in Barcelona, and both authors to the Isaac Newton Institute in Cambridge for their hospitality during the completion of the paper.

We thank Thomas Delzant, Denis Osin, and the anonymous referee for useful comments and corrections.

\section{Preliminaries on infinite small CANCEllation presentations}

A set of words $R$ in the alphabet $A$ is said to be symmetrized if it contains $r^{-1}$ and all the cyclic permutations of $r$ and $r^{-1}$, whenever $r \in R$. Without loss of generality we always assume that the set of group relators is symmetrized and that all relators $r \in R$ are reduced words in the alphabet $A$.

We focus on finitely generated groups with infinite presentations,

$$
G=\left\langle A \mid r_{1}, \ldots, r_{k}, \ldots\right\rangle,
$$

defined by a symmetrized family $R$ of relators consisting of an infinite sequence of relators $r_{1}, \ldots, r_{k}, \ldots$

We denote by $R_{k}$ the set $\left\{r_{1}, \ldots, r_{k}\right\}$ and by $G_{k}$ the finitely presented group

$$
G_{k}=\left\langle A \mid R_{k}\right\rangle=\left\langle A \mid r_{1}, \ldots, r_{k}\right\rangle .
$$

For two words $u, v$ we write $u \sqsubset v$ when $u$ is a subword of $v$. Let $\eta$ be a constant in $\left(0, \frac{1}{2}\right]$. If in the preceding we have moreover that

$$
\eta|v| \leqslant|u| \leqslant \frac{1}{2}|v|
$$

then we use the notation $u \sqsubset_{\eta} v$. We write $u \sqsubset R$ if there exists $v \in R$ such that $u \sqsubset v$. Similarly, for $\sqsubset$ replaced by $\sqsubset_{\eta}$.

Notation 2.1. We denote by $S(R)$ the set of words $u$ such that $u \sqsubset R$ and by $S^{\eta}(R)$ the set of words $u$ such that $u \sqsubset_{\eta} R$.

Definition $2.2\left(C^{\prime}(\lambda)\right.$-condition). Let $\lambda \in(0,1)$. A symmetrized set $R$ of words in the alphabet $A$ is said to satisfy the $C^{\prime}(\lambda)$-condition if the following holds:

(1) If $u$ is a subword in a word $r \in R$ so that $|u| \geqslant \lambda|r|$ then $u$ occurs only once in $r$;

(2) If $u$ is a subword in two distinct words $r_{1}, r_{2} \in R$ then $|u|<\lambda \min \left\{\left|r_{1}\right|,\left|r_{2}\right|\right\}$. We say that a group presentation $\langle A \mid R\rangle$ satisfies the $C^{\prime}(\lambda)$-condition if $R$ satisfies that condition.

Our technical arguments use the language of van Kampen diagrams over a group presentation $\langle A \mid R\rangle$, for more details and terminology see [LS77], and observe that the classical results below still hold for infinite group presentations.

The boundary of any van Kampen diagram (cell) $\Delta$ is denoted by $\partial \Delta$.

Lemma 2.3 (Greendlinger [LS77]Ch.V, Thm. 4.4). Every reduced van Kampen diagram $\Delta$ over the presentation (1) with small cancellation condition $C^{\prime}(\lambda)$ for $\lambda \leqslant \frac{1}{6}$ contains a cell $\Pi$ with $\partial \Pi$ labeled by a relator $r \in R$ such that $\partial \Delta \cap \partial \Pi$ has a connected component of length $>(1-3 \lambda)|r|$. 
Definition 2.4 ( $n$-gon). We call $n$-gon in a geodesic metric space a loop obtained by successive concatenation of $n$ geodesics.

We say that the $n$-gon is simple if the loop thus obtained is simple, that is, it does not have self-intersections.

Theorem 2.5 (cf. [GdlH91]). Let $\Delta$ be a reduced van Kampen diagram over a group presentation $G=\langle A \mid R\rangle$ satisfying the $C^{\prime}(\lambda)$-condition, with $\lambda \leqslant \frac{1}{8}$.

(1) Assume that $\partial \Delta$ is a simple bigon in the Cayley graph of $G$. Then it has the form of the bigon $B$ in Figure 1.

(2) Assume that $\partial \Delta$ is a simple triangle in the Cayley graph of $G$. Then it has one of the forms $T_{1}, \ldots, T_{4}$ in Figure 1 and Figure 2.
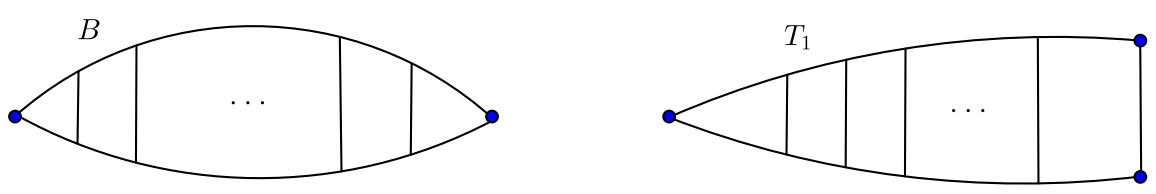

Figure 1. Simple bigon $B$ and simple triangle $T_{1}$.
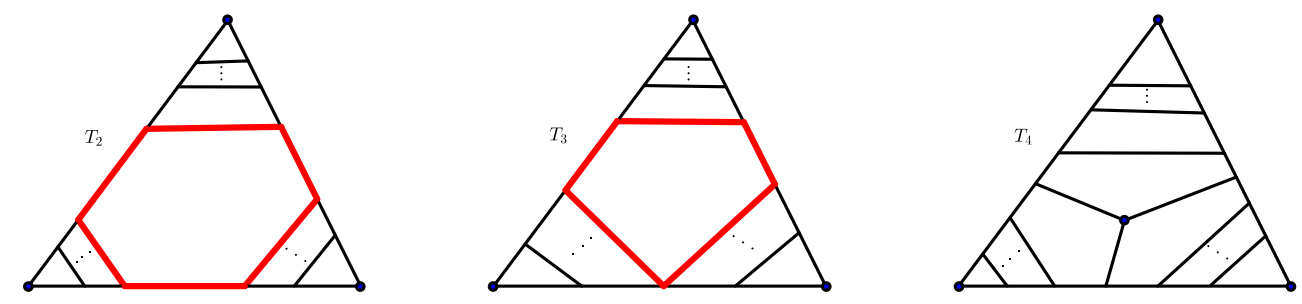

Figure 2. Simple triangles $T_{2}, T_{3}$, and $T_{4}$.

\section{Standard DECOMPosition of ELEMENTS IN SMALL CANCELlation GRoups}

This section is devoted to a thorough analysis of geodesics in Cayley graphs of infinitely presented small cancellation groups, and to the description of a set which, from many points of view, plays the part of the convex hull of a two points-set in irreducible buildings. We show here the main technical result of the paper, Theorem 3.15 , and its algebraic counterpart Theorem 3.27.

Convention 3.1. Throughout this section $G$ denotes a finitely generated group with a (possibly infinite) presentation $\langle A \mid R\rangle$ satisfying the $C^{\prime}(\lambda)$-condition with $\lambda \leqslant \frac{1}{8}$.

We only consider the Cayley graph of $G$ with respect to the fixed (arbitrary) finite generating set $A$, and we omit mentioning $A$ from now on. By "vertex" we shall always mean a vertex in that Cayley graph.

Copyright (c) 2018 Canadian Mathematical Society. All rights reserved. 
We call contour a loop in the Cayley graph of $G$ labeled by a relator $r \in R$. By abuse of notation, given a contour $t$ we denote by $|t|$ its length. Observe that a contour is always a simple loop (a non-trivial self-intersection leads to a contradiction with the small cancellation assumption by the Greendlinger lemma).

By an arc we mean a topological arc, that is the image of a topological embedding of an interval into a topological (in particular metric) space.

For every path $\mathfrak{p}$ in a metric space, we denote the initial point of $\mathfrak{p}$ by $\mathfrak{p}_{-}$and the terminal point of $\mathfrak{p}$ by $\mathfrak{p}_{+}$. Given two points $x, y$ on a geodesic $\mathfrak{g}$, we denote by $[x, y]$ the sub-geodesic of $\mathfrak{g}$ with endpoints $x, y$.

Lemma 3.2. Let $t$ be a contour labeled by a relator $r$ and let $a, b$ be two points on $t$.

(1) If one of the two arcs with endpoints $a, b$ has length $<\frac{|t|}{2}$ then that arc is the unique geodesic with endpoints $a, b$ in the Cayley graph.

(2) If both arcs with endpoints $a, b$ have length $\frac{|t|}{2}$ then these arcs are the only two geodesics with endpoints $a, b$ in the Cayley graph.

(3) The intersection of a geodesic with a contour is always composed of only one arc.

Proof. (1) Assume there exists a geodesic joining $a, b$ distinct from that arc. Then they compose at least one non-trivial simple bigon. Consider the minimal van Kampen diagram $\Delta$ with the same boundary label as this bigon. Let $u$ be the label of the sub-arc of $t$ and $v$ the label of the sub-arc of the geodesic. According to Lemma 2.3 , there exists a cell $\Pi$ labeled by a relator intersecting the boundary $\partial \Delta$ in an arc of length $>1-3 \lambda$ of the length of $\partial \Pi$.

Assume first that $\partial \Pi$ does not coincide with $t$. By the small cancellation condition, the arc can have at most $\lambda$ of the length of $\partial \Pi$ in common with the arc labeled by a subword of $r$, hence it has $>1-4 \lambda$ of the length of $\partial \Pi$ in common with the arc with the same label as the geodesic. As $\lambda \leqslant \frac{1}{8}$, this contradicts the fact that this is the label of a geodesic.

Now if $\partial \Pi$ coincides with $t$, then $\partial \Pi$ has at least $\frac{1}{2}-3 \lambda$ of its length in common with the arc labeled by $v$. In particular, it follows that $|u| \geqslant|v|>\left(\frac{1}{2}-3 \lambda\right)|r|$. Then $\partial \Delta \triangle \partial \Pi$ composes a new simple bigon with both sides of length at most $3 \lambda|r|$. We apply the argument above to this new bigon, the boundary of the cell provided by Lemma 2.3 cannot coincide with $t$ this time, and we obtain a contradiction.

(2) The argument to show that there exists no geodesic joining $a, b$ and which is not entirely contained in $t$ is as above.

(3) It suffices to prove that this intersection is path connected. Indeed, let $\mathfrak{g}$ be a geodesic and let $a, b$ be two points on $\mathfrak{g} \cap t$. The above arguments show that the part of $\mathfrak{g}$ between $a$ and $b$ must be contained in $t$.

Definition 3.3 (Relator-tied geodesics and components). Let $\mathfrak{g}$ be a geodesic in the Cayley graph of $G$ and let $\eta$ be a number in $(0,1)$.

(1) $\mathfrak{g}$ is called $\eta$-relator-tied if it is covered by sub-geodesics labeled by words in $S^{\eta}(R)$.

(2) an $\eta$-relator-tied component of $\mathfrak{g}$ is a maximal sub-geodesic of $\mathfrak{g}$ that is $\eta$-relator-tied.

Lemma 3.4. (1) The $\eta$-relator-tied components of a geodesic $\mathfrak{g}$ are disjoint. 
(2) Assume that $\eta \leqslant \frac{1}{2}-2 \lambda$. If two points $a, b$ are the endpoints of a geodesic $\mathfrak{g}$ with no $\eta$-relator-tied component then $\mathfrak{g}$ is the unique geodesic with endpoints $a, b$.

Proof. Assertion (1) follows by definition, since two distinct $\eta$-relator-tied subgeodesics that intersect compose a longer $\eta$-relator-tied sub-geodesic.

(2) Any other geodesic $\mathfrak{g}^{\prime}$ with endpoints $a, b$ and distinct from $\mathfrak{g}$ would compose with $\mathfrak{g}$ simple geodesic bigons, therefore by Theorem $2.5,(1), \mathfrak{g}$ would contain a $\left(\frac{1}{2}-2 \lambda\right)$-relator-tied component.

Definition 3.5 ( $\eta$-compulsory geodesic). Given $0<\eta \leqslant \frac{1}{2}-2 \lambda$, a geodesic as in Lemma 3.4, (2), is called an $\eta$-compulsory geodesic. A pair of endpoints $a, b$ of an $\eta$-compulsory geodesic is called an $\eta$-compulsory pair.

We now proceed to analyze the $\eta$-relator-tied components of geodesics.

Lemma 3.6. Let $\eta \geqslant 2 \lambda$. Let $\mathfrak{g}$ be a $\eta$-relator-tied geodesic in the Cayley graph of $G$. Then there exists a unique sequence of successive vertices

$$
x_{0}=a, x_{1}, y_{0}, x_{2}, y_{1}, \ldots, x_{k+1}, y_{k}, y_{k+1}=b
$$

such that the sub-geodesics with endpoints $x_{i}, y_{i}$ with $i \in\{0,1, \ldots, k+1\}$ are labeled by words in $S^{\eta}(R)$, and are maximal with this property with respect to inclusion (see Figure 3).

Proof. By hypothesis $\mathfrak{g} \subseteq \bigcup_{i \in S_{0}} \mathfrak{g}_{i}$, where $\mathfrak{g}_{i}$ denotes a sub-geodesic of $\mathfrak{g}$ labeled by a word $u_{i} \in S^{\eta}(R)$ and the index set $S_{0}$ is finite (by compactness).

Without loss of generality, we assume that all the sub-geodesics $\mathfrak{g}_{i}$ in the covering above are maximal with respect to inclusion.

Indeed, we begin by the sub-geodesics containing the vertex $\mathfrak{g}_{-}$. Consider two such sub-geodesics. If one is contained in the other, by the $C^{\prime}(\lambda)$-condition and the fact that $\eta \geqslant 2 \lambda$ it follows that both are subwords of the same relator $r_{1} \in R$. Therefore we take the longer of the two subwords and we select it as the first term $\mathfrak{g}_{1}$ of the new covering. The endpoint $\left(\mathfrak{g}_{1}\right)_{+}$must be contained in another sub-geodesic $\mathfrak{g}_{u}$. The sub-geodesic $\mathfrak{g}_{1} \sqcup \mathfrak{g}_{u}$ cannot be labeled by a word in $S^{\eta}(R)$, because this would contradict the maximality of $\mathfrak{g}_{1}$. We consider the maximal sub-geodesic $\mathfrak{g}_{2}$ labeled by a word in $S^{\eta}(R)$ and containing $\mathfrak{g}_{u}$. Continuing this argument, we obtain a cover $\bigcup_{i \in S_{1}} \mathfrak{g}_{i}$ of $\mathfrak{g}$ for some $S_{1} \subseteq S_{0}$ such that $\mathfrak{g}_{i}$ are maximal sub-geodesics labeled by words in $S^{\eta}(R)$.

For an arbitrary small $\varepsilon>0$ we have that $\mathfrak{g} \subseteq \bigcup_{i \in S_{1}} \mathfrak{g}_{i}^{\varepsilon}$, where $\mathfrak{g}_{i}^{\varepsilon}$ denotes the $\varepsilon$-neighborhood of $\mathfrak{g}_{i}$ in $\mathfrak{g}$. Since $\mathfrak{g}$ has topological dimension one, there exists $S_{2} \subseteq S_{1}$ such that $\mathfrak{g} \subseteq \bigcup_{i \in S_{2}} \mathfrak{g}_{i}^{\varepsilon}$ and every point in $\mathfrak{g}$ is contained in at most two sets $\mathfrak{g}_{i}^{\varepsilon}$ with $i \in S_{2}$.

If an edge $e$ in $\mathfrak{g}$ is not contained in $\bigcup_{i \in S_{2}} \mathfrak{g}_{i}$ then for $\varepsilon<\frac{1}{2}$ this contradicts the fact that $\left\{\mathfrak{g}_{i}^{\varepsilon} \mid i \in S_{2}\right\}$ cover $e$. If a vertex in $\mathfrak{g}$ is not contained in $\bigcup_{i \in S_{2}} \mathfrak{g}_{i}$, then the edges adjacent to it are not contained in $\bigcup_{i \in S_{2}} \mathfrak{g}_{i}$ and we use the above.

We thus obtain that $\mathfrak{g} \subseteq \bigcup_{i \in S_{2}} \mathfrak{g}_{i}$ and every point in $\mathfrak{g}$ is contained in at most two sets $\mathfrak{g}_{i}$ with $i \in S_{2}$.

Assume that there exist two sequences $x_{0}=a, x_{1}, y_{0}, x_{2}, y_{1}, \ldots, x_{k+1}, y_{k}, y_{k+1}=$ $b$ and $x_{0}^{\prime}=a, x_{1}^{\prime}, y_{0}^{\prime}, x_{2}^{\prime}, y_{1}^{\prime}, \ldots, x_{m+1}^{\prime}, y_{m}^{\prime}, y_{m+1}^{\prime}=b$, and let $k \leqslant m$. We prove by induction on $0 \leqslant i \leqslant k+1$ that $\left[x_{i}, y_{i}\right]=\left[x_{i}^{\prime}, y_{i}^{\prime}\right]$.

Copyright (c) 2018 Canadian Mathematical Society. All rights reserved. 
First, consider case $i=0$. Then either $\left[x_{0}, y_{0}\right] \subseteq\left[x_{0}^{\prime}, y_{0}^{\prime}\right]$ or $\left[x_{0}^{\prime}, y_{0}^{\prime}\right] \subseteq\left[x_{0}, y_{0}\right]$. The assumption $\eta \geqslant 2 \lambda$ implies that both the label of $\left[x_{0}, y_{0}\right]$ and that of $\left[x_{0}^{\prime}, y_{0}^{\prime}\right]$ are subwords of the same relator $r$. The maximality condition implies that $\left[x_{0}, y_{0}\right]=$ $\left[x_{0}^{\prime}, y_{0}^{\prime}\right]$.

We now assume that for some $j \geqslant 0$ we have $\left[x_{i}, y_{i}\right]=\left[x_{i}^{\prime}, y_{i}^{\prime}\right]$ for $0 \leqslant i \leqslant j$. We have that either $\left[y_{j}, y_{j+1}\right] \subseteq\left[y_{j}, y_{j+1}^{\prime}\right]$ or $\left[y_{j}, y_{j+1}^{\prime}\right] \subseteq\left[y_{j}, y_{j+1}\right]$. By maximality and Lemma 3.2 , the contour $t_{j}$ containing the geodesic $\left[x_{j}, y_{j}\right]$ is distinct from the contour $t_{j+1}$ containing the geodesic $\left[x_{j+1}, y_{j+1}\right]$, respectively the contour $t_{j+1}^{\prime}$ containing the geodesic $\left[x_{j+1}^{\prime}, y_{j+1}^{\prime}\right]$, see Figure 4 . It follows that

$$
\operatorname{dist}\left(x_{j+1}, y_{j}\right)<\lambda\left|r_{j+1}\right| \leqslant \frac{\lambda}{\eta} \operatorname{dist}\left(x_{j+1}, y_{j+1}\right),
$$

whence

$$
\operatorname{dist}\left(y_{j}, y_{j+1}\right)>\left(1-\frac{\lambda}{\eta}\right) \operatorname{dist}\left(x_{j+1}, y_{j+1}\right) \geqslant \eta\left(1-\frac{\lambda}{\eta}\right)\left|r_{j+1}\right| .
$$

Similarly, we obtain that

$$
\operatorname{dist}\left(y_{j}, y_{j+1}^{\prime}\right)>\eta\left(1-\frac{\lambda}{\eta}\right)\left|r_{j+1}^{\prime}\right| .
$$

The hypothesis $\eta \geqslant 2 \lambda$ implies that $\eta\left(1-\frac{\lambda}{\eta}\right) \geqslant \lambda$, therefore the inclusions

$$
\left[y_{j}, y_{j+1}\right] \subseteq\left[y_{j}, y_{j+1}^{\prime}\right] \text { or }\left[y_{j}, y_{j+1}^{\prime}\right] \subseteq\left[y_{j}, y_{j+1}\right]
$$

imply that $t_{j+1}=t_{j+1}^{\prime}$. The maximality of the sub-geodesics $\left[x_{j+1}, y_{j+1}\right]$ and $\left[x_{j+1}^{\prime}, y_{j+1}^{\prime}\right]$, and Lemma 3.2 allow to conclude that $\left[x_{j+1}, y_{j+1}\right]=\left[x_{j+1}^{\prime}, y_{j+1}^{\prime}\right]$.

Convention 3.7. For the rest of this section, let $\eta$ be a fixed constant such that $\frac{1}{2}-2 \lambda \geqslant \eta \geqslant 2 \lambda$ and $\eta^{\prime}:=\eta-\lambda$.

Definition 3.8 ( $\eta$-succession). We say that a sequence of contours $t_{0}, t_{1}, \ldots, t_{k+1}$ is an $\eta$-succession of contours if, for every $i$, one of the endpoints of $t_{i-1} \cap t_{i}$ is at distance $\geqslant \eta\left|t_{i}\right|$ from at least one of the endpoints of $t_{i} \cap t_{i+1}$ (distance measured in $t_{i}$ ).

Corollary 3.9. Let $\mathfrak{g}$ be an $\eta$-relator-tied geodesic. Then there exists a unique $\eta$-succession of contours $t_{0}, t_{1}, \ldots, t_{k+1}$ such that for the decomposition described in Lemma 3.6 the sub-geodesic with endpoints $x_{i}, y_{i}$ is contained in $t_{i}$.

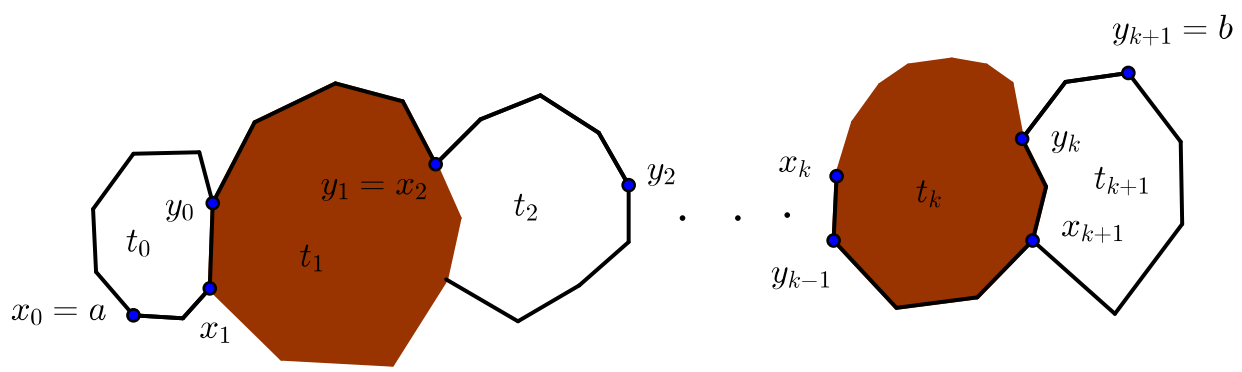

FiguRE 3. An $\eta$-relator-tied geodesic inside a succession of contours. 


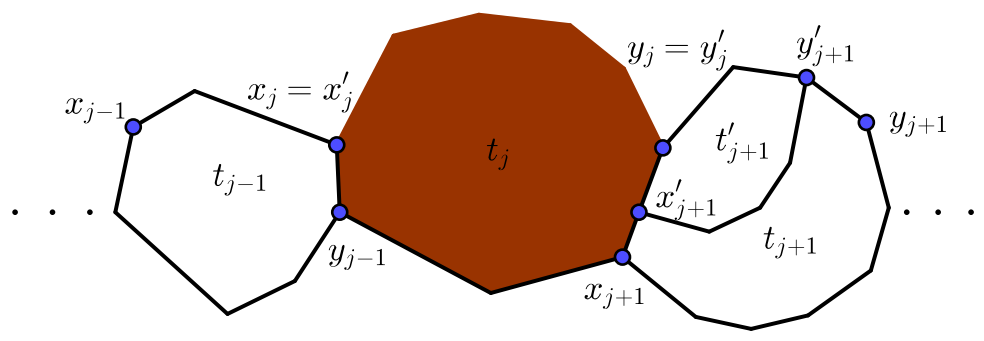

Figure 4. The uniqueness of the decomposition in Lemma 3.6.

Lemma 3.10. Let $a, b$ be two vertices joined by an $\eta$-relator-tied geodesic $\mathfrak{g}$. Then every geodesic $\mathfrak{g}^{\prime}$ with endpoints a, $b$ is $\eta^{\prime}$-relator-tied, for $\eta^{\prime}:=\eta-\lambda$; moreover, $\mathfrak{g}^{\prime}$ is contained in the $\eta$-succession of contours $t_{0}, t_{1}, \ldots, t_{k+1}$ determined by $\mathfrak{g}$ according to Corollary 3.9.

Proof. There exist successive points in the intersection $\mathfrak{g} \cap \mathfrak{g}^{\prime}$,

$$
z_{0}=a, z_{1}, \ldots, z_{2 m-1}, z_{2 m}, z_{2 m+1}=b
$$

such that $z_{2 i}, z_{2 i+1}$ are the endpoints of a connected component of $\mathfrak{g} \cap \mathfrak{g}^{\prime}$, while $z_{2 i+1}, z_{2 i+2}$ are the endpoints of two sub-geodesics of $\mathfrak{g}$ respectively $\mathfrak{g}^{\prime}$, composing a simple bigon.

Let $x_{0}=a, x_{1}, y_{0}, x_{2}, y_{1}, \ldots, x_{k+1}, y_{k}, y_{k+1}=b$ be the unique sequence of points on $\mathfrak{g}$ provided by Lemma 3.6. For every $0 \leqslant i \leqslant m-1$ consider the endpoints $z_{2 i+1}, z_{2 i+2}$ of a simple bigon. According to Theorem 2.5, (1), the corresponding bigon is as in Figure 1. Consider $\tau$, one of the contours appearing in this bigon; let $\alpha, \beta$ be the endpoints of the intersection of $\mathfrak{g}$ with $\tau$. By the small cancellation condition and the fact that the two sides of the bigon are geodesics it follows that the label of the sub-geodesic of $\mathfrak{g}$ limited by $\alpha, \beta$ is a sub-word of length $>\left(\frac{1}{2}-2 \lambda\right)|\tau|$.

Let $m$ be the midpoint of the sub-geodesic of $\mathfrak{g}$ limited by $\alpha, \beta$. Then there exist $x_{j}, y_{j}$ separated by $m$. If the contour $\tau$ is distinct from the contour $t_{j}$ then $\frac{1}{2}\left(\frac{1}{2}-2 \lambda\right)|\tau|<\frac{1}{2} \operatorname{dist}(\alpha, \beta)<\lambda|\tau|$, whence $\lambda>\frac{1}{8}$, a contradiction. It follows that $\tau=t_{j}$, hence $\alpha=x_{j}$ and $\beta=y_{j}$. Thus, the endpoints of intersections of contours of the bigon with $\mathfrak{g}$ compose a subsequence of the sequence $x_{0}=a, x_{1}, y_{0}, x_{2}, y_{1}, \ldots, x_{k+1}, y_{k}, y_{k+1}=b$, with the property that $x_{i+1}=y_{i}$.

Let $z_{2 i+1}$ be an endpoint of a bigon. According to the above $z_{2 i+1}$ equals some $x_{j}$ such that $t_{j}$ is the first contour in the bigon. Consider now $x_{j-1}, y_{j-1}$ and the contour $t_{j-1} \neq t_{j}$. Then $\operatorname{dist}\left(x_{j}, y_{j-1}\right)<\lambda\left|t_{j-1}\right|$, whence $\operatorname{dist}\left(x_{j-1}, x_{j}\right)=$ $\operatorname{dist}\left(x_{j-1}, y_{j-1}\right)-\operatorname{dist}\left(x_{j}, y_{j-1}\right)>\eta\left|t_{j-1}\right|-\lambda\left|t_{j-1}\right|=\eta^{\prime}\left|t_{j-1}\right|$.

We thus found that the sub-geodesic with endpoints $z_{2 i}, z_{2 i+1}$ common to $\mathfrak{g}$ and $\mathfrak{g}^{\prime}$ is $\eta^{\prime}$-relator-tied. A sub-geodesic of $\mathfrak{g}^{\prime}$ composing one of the simple bigons is easily seen to be $\eta^{\prime}$-relator-tied as $\eta^{\prime} \leqslant \frac{1}{2}-2 \lambda$, hence the entire of $\mathfrak{g}^{\prime}$ is $\eta^{\prime}$-relatortied.

The fact that $\mathfrak{g}^{\prime}$ is contained in the $\eta$-succession of contours $t_{0}, t_{1}, \ldots, t_{k+1}$ is immediate from the argument above: the sub-arcs of $\mathfrak{g}^{\prime}$ with endpoints $z_{2 i}, z_{2 i+1}$ are contained in $\mathfrak{g}$, while the sub-arcs with endpoints $z_{2 i+1}, z_{2 i+2}$ are covered by contours $\tau$ which are in the set $\left\{t_{0}, t_{1}, \ldots, t_{k+1}\right\}$. 
The goal of the following two statements is to prepare the ground for the definition of the $\eta$-criss-cross decomposition for a pair of vertices $a, b$.

Lemma 3.11. Let $a$ and $b$ be two arbitrary vertices. The endpoints of an $\eta$-relatortied component in a geodesic joining $a, b$ are contained in any other geodesic joining $a, b$.

Proof. Let $\mathfrak{g}, \mathfrak{g}^{\prime}$ be two geodesics with endpoints $a, b$ and let $x, y$ be the endpoints of an $\eta$-relator-tied component on $\mathfrak{g}$. Assume that $x$ is not on $\mathfrak{g}^{\prime}$. Then $x$ is in the interior of one of the sides of a bigon composed by $\mathfrak{g}$ and $\mathfrak{g}^{\prime}$. On the other hand, this side is $\left(\frac{1}{2}-2 \lambda\right)$-relator-tied, hence the component of $\mathfrak{g}$ between $x, y$ is not a maximal $\eta$-relator-tied sub-geodesic, a contradiction.

It follows that $x \in \mathfrak{g}^{\prime}$ and a similar argument shows that $y \in \mathfrak{g}^{\prime}$.

Definition 3.12 (Geodesic sequences). (1) We say that a vertex $p$ is between two vertices $a$ and $b$ if $\operatorname{dist}(a, p)+\operatorname{dist}(p, b)=\operatorname{dist}(a, b)$. We do not exclude that $p=a$ or $p=b$.

(2) We call geodesic sequence a finite sequence of vertices $p_{1}, \ldots, p_{m}$ such that for every $1 \leqslant i \leqslant j \leqslant k \leqslant m, p_{j}$ is between $p_{i}$ and $p_{k}$.

(3) If $a, b, c, d$ is a geodesic sequence then we write $(b, c) \Subset(a, d)$ and we say that the pairs $(b, c)$ and $(a, d)$ are nested.

Lemma 3.13. Let $p, a, q, b$ be a geodesic sequence such that $p, q$ and respectively $a, b$ are the endpoints of $\eta$-relator-tied geodesics. Then there exists an $\eta$-succession of contours that contains every geodesic joining $p$ and $b$.

Proof. We denote by $[p, q]$ and respectively $[a, b]$ the $\eta$-relator-tied geodesics. Consider two arbitrary geodesics $[p, a]$ and $[q, b]$ (not necessarily contained in $[p, q]$ and respectively $[a, b])$.

In the geodesic $[p, a] \cup[a, b]$, the sub-geodesic $[a, b]$ is contained in a maximal $\eta$ relator-tied component $\left[a^{\prime}, b\right]$. Lemma 3.11 applied to $p, b$ and the geodesic joining them $[p, q] \cup[q, b]$ implies that $a^{\prime} \in[p, q]$, moreover $a^{\prime}$ is on every geodesic joining $p, b$. Thus, by possibly replacing $a$ with $a^{\prime}$ we may assume that $a$ is contained in every geodesic with endpoints $p, b$, in particular that $a \in[p, q]$. A similar argument allows to state that without loss of generality we may assume that $q$ is contained in every geodesic joining $p, b$, in particular $q \in[a, b]$.

By Corollary 3.9, there exist two $\eta$-successions of contours,

$$
t_{0}, t_{1}, \ldots, t_{k+1} \text { and } \tau_{0}, \tau_{1}, \ldots, \tau_{m+1}
$$

such that every geodesic joining $p, q$ is contained in $\bigcup_{i=0}^{k+1} t_{i}$, and every geodesic joining $a, b$ is contained in $\bigcup_{j=0}^{m+1} \tau_{j}$.

Consider $i$ maximal such that $a \in t_{i}$.

Assume $i \neq k+1$. If $\tau_{0} \neq t_{i}$ then $[a, b] \cap \tau_{0}$ intersects $t_{i}$ in a sub-geodesic of length $<\lambda\left|\tau_{0}\right|$, consequently it intersects $t_{i+1}$ in a sub-geodesic of length either at least $\lambda\left|\tau_{0}\right|$ or at least $(\eta-\lambda)\left|t_{i+1}\right|$. In both cases it follows $\tau_{0}=t_{i+1}$, whence $a \in t_{i+1}$, which contradicts the choice of $i$.

Thus, in this case, it follows that $\tau_{0}=t_{i}$.

Let $\ell \geqslant 0$ be maximal such that $\tau_{r}=t_{i+r}$ for $0 \leqslant r \leqslant \ell$. It is immediate from the definition of an $\eta$-succession that the sequence

$$
t_{0}, \ldots, t_{i}=\tau_{0}, \ldots, t_{i+\ell}=\tau_{\ell}, \tau_{\ell+1}, \ldots, \tau_{m+1}
$$

Copyright (c) 2018 Canadian Mathematical Society. All rights reserved. 
is such a succession.

An arbitrary geodesic joining $p$ and $b$ must contain $a$ and $q$, the sub-geodesic from $p$ to $a$ must be contained in $\bigcup_{j=0}^{i} t_{j}$, while the sub-geodesic from $a$ to $b$ must be contained in $\bigcup_{r=0}^{m+1} \tau_{r}$.

Assume now that $i=k+1$. Every geodesic joining $a, q$ must be contained in $t_{k+1}$.

Suppose moreover that $\tau_{0} \neq t_{k+1}$. Then $\operatorname{dist}(a, q)<\lambda \min \left\{\left|t_{k+1}\right|,\left|\tau_{0}\right|\right\}$. Since the distance from $q$ to one of the endpoints of $t_{k} \cap t_{k+1}$ is at least $\eta\left|t_{k+1}\right|$, the same is true about one of the endpoints of $\tau_{0} \cap \tau_{1}$, since it will be situated after $q$ on a geodesic from $a$ to $b$. Therefore, in this case

$$
t_{0}, t_{1}, \ldots, t_{k+1}, \tau_{0}, \tau_{1}, \ldots, \tau_{m+1}
$$

is an $\eta$-succession of contours.

Given an arbitrary geodesic joining $p$ and $b$, the sub-geodesic from $p$ to $q$ is in $\bigcup_{j=0}^{k+1} t_{j}$, the sub-geodesic from $a$ to $b$ is in $\bigcup_{r=0}^{m+1} \tau_{r}$.

Suppose that $\tau_{0}=t_{k+1}$. As before, the fact that $q$ is at distance $\geqslant \eta\left|\tau_{0}\right|$ from one of the endpoints of $t_{k} \cap t_{k+1}$ implies that one of the endpoints of $\tau_{0} \cap \tau_{1}$ satisfies the same. Therefore,

$$
t_{0}, t_{1}, \ldots, t_{k+1}=\tau_{0}, \tau_{1}, \ldots, \tau_{m+1}
$$

is an $\eta$-succession of contours, and an argument as above shows that it contains every geodesic joining $p$ and $b$.

Remark 3.14. The statement of Lemma 3.13 can be generalized as follows: if

$$
p_{0}, p_{1}, q_{0}, p_{2}, q_{1}, \ldots, p_{k+1}, q_{k}, q_{k+1}
$$

is a geodesic sequence such that $p_{i}, q_{i}$ are the endpoints of $\eta$-relator tied geodesics for $i \in\{0,1, \ldots, k+1\}$, then there exists an $\eta$-succession of contours containing every geodesic from $p_{0}$ to $q_{k+1}$.

The proof adapts the argument of Lemma 3.13, and we leave it as an exercise to the reader.

Theorem 3.15. For every pair of vertices a, $b$ in the Cayley graph of $G$ there exists a finite geodesic sequence

$$
z_{0}=a, y_{1}, z_{1}, y_{2}, z_{2}, \ldots, y_{m}, z_{m}, b=y_{m+1},
$$

a sequence of $\eta$-compulsory geodesics $\left[z_{0}, y_{1}\right],\left[z_{1}, y_{2}\right], \ldots,\left[z_{i}, y_{i+1}\right], \ldots,\left[z_{m}, y_{m+1}\right]$ and a sequence of $\eta$-successions of contours

$$
t_{1}^{(i)}, \ldots, t_{k_{i}}^{(i)}, i \in\{1,2, \ldots, m\}
$$

such that $y_{i} \in t_{1}^{(i)}, z_{i} \in t_{k_{i}}^{(i)}$ and every geodesic joining $a, b$ is contained in

$$
\left[a, y_{1}\right] \cup \bigcup_{j=1}^{k_{1}} t_{j}^{(1)} \cup\left[z_{1}, y_{2}\right] \cup \bigcup_{j=1}^{k_{2}} t_{j}^{(2)} \cup \cdots \cup\left[z_{i-1}, y_{i}\right] \cup \bigcup_{j=1}^{k_{i}} t_{j}^{(i)} \cup\left[z_{i}, y_{i+1}\right] \cup \cdots \cup\left[z_{m}, b\right]
$$

Definition 3.16 ( $\eta$-criss-cross decomposition). We say that the sequence

$$
\left(a, y_{1}\right), \backslash y_{1}, z_{1} /,\left(z_{1}, y_{2}\right), \backslash y_{2}, z_{2} /, \ldots, \backslash y_{m}, z_{m} /,\left(z_{m}, b\right)
$$

is the $\eta$-criss-cross decomposition for the pair $a, b$. 


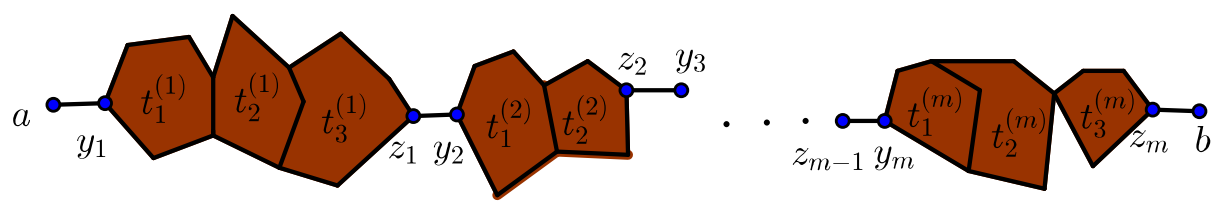

Figure 5 . The $\eta$-criss-cross decomposition for the pair $a, b$.

Notation 3.17. For an arbitrary pair of vertices $a, b$ we denote by $\mathcal{G}^{\eta}(a, b)$ the set described in Theorem 3.15, see (3) and Figure 5.

Proof of Theorem 3.15. If $a, b$ is an $\eta$-compulsory pair then there is nothing to prove. Assume therefore that there exists a geodesic joining $a, b$ with an $\eta$-relatortied component. Let $p_{1}, q_{1}, \ldots, p_{h}, q_{h}$ be all the pairs of points that appear as endpoints of $\eta$-relator-tied components in some geodesic joining $a, b$. Let $\mathfrak{g}$ be an arbitrary geodesic joining $a, b$. According to Lemma $3.11, \mathfrak{g}$ contains all points $p_{1}, q_{1}, \ldots, p_{h}, q_{h}$. The order in which these points appear is independent of the choice of $\mathfrak{g}$, since it is only determined by metric relations.

We consider the union $\bigcup_{i=1}^{h}\left[p_{i}, q_{i}\right]$, where $\left[p_{i}, q_{i}\right]$ denotes here the sub-geodesic of $\mathfrak{g}$ with endpoints $p_{i}, q_{i}$. The connected components of this union are sub-geodesics $\left[y_{1}, z_{1}\right], \ldots,\left[y_{m}, z_{m}\right]$ appearing on $\mathfrak{g}$ in this order. Note that $y_{i} \in\left\{p_{1}, \ldots, p_{h}\right\}$ and that $z_{i} \in\left\{q_{1}, \ldots, q_{h}\right\}$. In particular, both the points and the order are independent of the choice of the geodesic $\mathfrak{g}$.

It remains to apply Lemma 3.13 and Remark 3.14.

Corollary 3.18. For every pair of points $a, b$ at distance $d>0$ and every $x \leqslant d$ there exist at most 2 points $p$ with the property that $a, p, b$ is a geodesic sequence and $\operatorname{dist}(a, p)=x$.

See Figure 6 for an example where there exist two points $q_{1}, q_{2}$ between $a$ and $b$, at distance $x-3$ from $a$, and two points $p_{1}, p_{2}$ between $a$ and $b$, at distance $x$ from $a$.

Remark 3.19. Note that

(1) according to the above every geodesic with endpoints $y_{m}, z_{m}$ is $\eta^{\prime}$-relatortied, in particular, it is non-trivial;

(2) due to the maximality condition defining the pairs $y_{i}, z_{i}$, we have that $z_{i} \neq y_{i+1}$ for every $1 \leqslant i \leqslant m-1$

(3) on the other hand, in the pairs $\left(a, y_{1}\right),\left(z_{m}, b\right)$ the endpoints may coincide.

Remark 3.20. If $(p, q) \Subset(x, y) \Subset(a, b)$ with $p, q, x, y$ points in $\left\{p_{1}, q_{1}, \ldots, p_{k}, q_{k}\right\}$, and if $p, q$ are endpoints of an $\eta$-relator-tied component in a geodesic joining $a, b$, then $p, q$ are endpoints of an $\eta$-relator-tied component in a geodesic joining $x, y$.

This simply follows from the fact that a geodesic $\mathfrak{g}$ joining $a, b$ and on which $p, q$ bound an $\eta$-relator-tied component must also contain $x, y$, see Lemma 3.11.

Copyright (c) 2018 Canadian Mathematical Society. All rights reserved. 


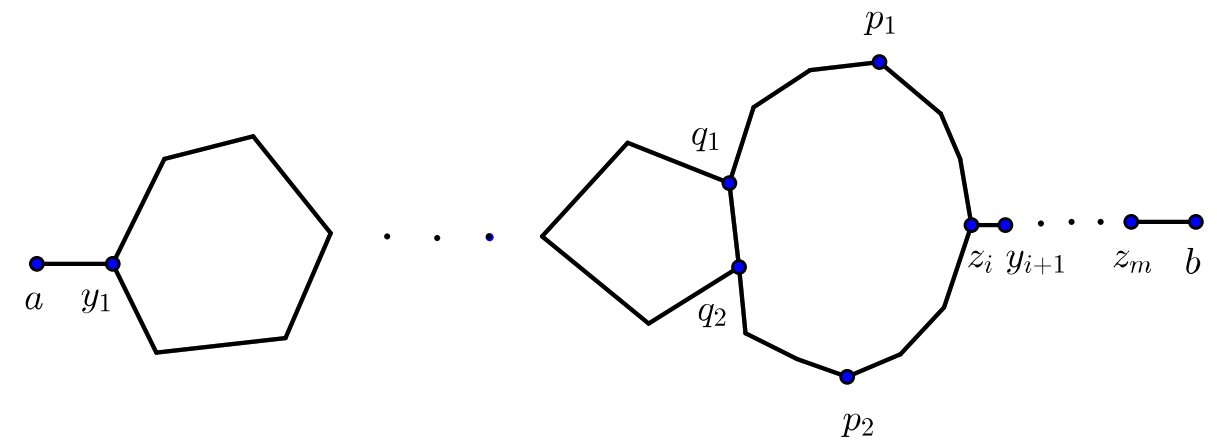

Figure 6. Example of pair $a, b$ with two points between them at distance $x$ from $a$.

Definition 3.21 ( $\eta$-relator covered pair). If the $\eta$-criss-cross decomposition of a pair $a, b$ is $\backslash a, b /$, then we call such a pair an $\eta$-relator covered pair.

Definition 3.22 (Compulsory vertices). Given an $\eta$-criss-cross decomposition

$$
\left(a, y_{1}\right), \backslash y_{1}, z_{1} /,\left(z_{1}, y_{2}\right), \backslash y_{2}, z_{2} /, \ldots, \backslash y_{m}, z_{m} /,\left(z_{m}, b\right)
$$

of a pair $a, b$, we call the vertices between $z_{i}, y_{i+1}$ for some $i \in\{1,2, \ldots, m-1\}$ $\eta$-compulsory vertices.

Clearly, every geodesic with endpoints $a$ and $b$ must contain all the compulsory vertices.

Definition 3.23 (Prefixes and suffixes). Given an element $h \in G$ we denote by $P(h)$ (standing for prefixes of $h$ ) all the elements between $1, h$ and by $S(h)$ (standing for suffixes of $h$ ) all the elements of the form $x^{-1} h$ for $x \in P(h)$.

Note that the two sets $P(h)$ and $S(h)$ depend on the fixed generating set $A$.

Definition 3.24 (Compulsory and $\eta$-relator-covered elements). Let $h \in G$.

- If $h$ is joined to 1 by at least one $\eta$-relator-tied geodesic then we call $h$ an $\eta$-relator-tied element.

- If the pair $1, h$ has the $\eta$-criss-cross decomposition $(1, h)$ (hence, there exists only one geodesic joining $1, h$, composed of compulsory vertices), then we call $h$ an $\eta$-compulsory element.

- If the pair $1, h$ has the $\eta$-criss-cross decomposition $\backslash 1, h /$ then we call $h$ an $\eta$-relator-covered element.

Notation 3.25. We denote by $R T^{\eta}$ the set of $\eta$-relator-tied elements. We denote by $\mathcal{C}^{\eta}$ the set of $\eta$-compulsory elements in $G$ and by $R C^{\eta}$ the set of $\eta$-relator-covered elements.

Remark 3.26. The fact that $h$ is $\eta$-relator-covered does not mean that there exists an $\eta$-relator-tied geodesic labeled by $h$, it only means that every geodesic $[a, b]$ labeled by $h$ contains a family of successive vertices $y_{0}=a, y_{1}, z_{0}, y_{2}, z_{1}, \ldots$, $y_{m}, z_{m-1}, z_{m}=b$ such that for every $i$ there exists an $\eta$-relator-tied geodesic with 
endpoints $y_{i}, z_{i}$. In particular, by Lemma 3.10, every geodesic labeled by $h$ is $\eta^{\prime}$-relator-tied.

An algebraic version of Theorem 3.15 is the following.

Theorem 3.27. Every element $g \in G$ can be written uniquely as a product

$$
g=\alpha_{1} \beta_{1} \alpha_{2} \beta_{2} \ldots \alpha_{m} \beta_{m} \alpha_{m+1}
$$

such that

- $\beta_{i}$ are non-trivial $\eta$-relator-covered elements;

- $\alpha_{i}$ are compulsory elements (non-trivial with the possible exception of $\alpha_{1}$, $\left.\alpha_{m+1}\right)$;

- the vertices $y_{i}=\alpha_{1} \beta_{1} \ldots \alpha_{i}$ and $z_{i}=\alpha_{1} \beta_{1} \ldots \alpha_{i} \beta_{i}$ compose the geodesic sequence determining the $\eta$-criss-cross decomposition of the pair $1, g$.

Notation 3.28. Given an arbitrary element $h \in G$ we denote by $\mathcal{G}^{\eta}(h)$ the set $\mathcal{G}^{\eta}(1, h)$ as described in Notation 3.17 .

Notation 3.29. Given $i \in \mathbb{N}, i \geqslant 2$, we denote by $\mathcal{D}_{i}$ the set of $i$-tuples

$$
\left(a_{1}, a_{2}, \ldots, a_{i-1}, b\right)
$$

such that for the element $g=a_{1} a_{2} \cdots a_{i-1} b$ the elements $a_{1}, a_{2}, \ldots, a_{i-1}$ are the first $i-1$ elements in the criss-cross decomposition of $g$ as described in Theorem $3.2 \%$.

The following lemma will be crucial for the results in Section 4 on quasi-homomorphisms.

Lemma 3.30. Let $\lambda \leqslant \frac{1}{10}$ and let $\eta \geqslant 3 \lambda$.

Every $\eta$-succession of contours $t_{0}, t_{1}, \ldots, t_{k+1}$ is totally geodesic: if $a, b$ are two vertices in $\bigcup_{i=0}^{k+1} t_{i}$ then every geodesic joining $a$ and $b$ is contained in $\bigcup_{i=0}^{k+1} t_{i}$.

Proof. Without loss of generality we assume that $a \in t_{1} \backslash t_{2}$ and that $b \in t_{k+1} \backslash t_{k}$. Otherwise, assuming that $a$ appears before $b$ in the succession, we consider the largest $i$ such that $t_{i}$ contains $a$ and the smallest $j$ such that $t_{j}$ contains $b$ and take the succession $t_{i}, t_{i+1}, \ldots, t_{j-1}, t_{j}$ instead of the initial one.

Let $\mathfrak{g}$ be a geodesic joining $a$ and $b$. We argue for a contradiction and assume that $\mathfrak{g}$ is not contained in $\bigcup_{i=0}^{k+1} t_{i}$. Without loss of generality we assume that $\mathfrak{g}$ intersects $\bigcup_{i=0}^{k+1} t_{i}$ only in its endpoints (otherwise, we replace $\mathfrak{g}$ by a sub-geodesic with this property).

Let $\mathfrak{p}$ be a topological arc joining $a$ and $b$ in $\bigcup_{i=0}^{k+1} t_{i}$ and of minimal length. By the Greendlinger Lemma, there exists a contour $\tau$ such that one of the connected components of its intersection with $\mathfrak{p} \cup \mathfrak{g}$ has length $>(1-3 \lambda)|\tau|$. If $\tau=t_{i}$ for some $i$ then by the hypothesis on $\mathfrak{g}, \tau$ intersects $\mathfrak{p}$ in a connected component of length $>(1-3 \lambda)|\tau|$. Then $\mathfrak{p}$ can be shortened by a length of $(1-6 \lambda)|\tau|$, which contradicts the choice of $\mathfrak{p}$ as an arc of minimal length joining $a$ and $b$ in $\bigcup_{i=0}^{k+1} t_{i}$.

We therefore assume that $\tau \notin\left\{t_{0}, t_{1}, \ldots, t_{k+1}\right\}$. Since $\mathfrak{g}$ is a geodesic, it follows that $\tau$ intersects $\mathfrak{p}$ in a subarc of length $>\left(\frac{1}{2}-3 \lambda\right)|\tau|$.

On the other hand, $\mathfrak{p}$ contains a succession of vertices

$$
x_{0}=a, x_{1}, y_{0}, x_{2}, y_{1}, \ldots, x_{k+1}, y_{k}, y_{k+1}=b
$$

such that the sub-arcs with endpoints $x_{i}, y_{i}$ with $i \in\{0,1, \ldots, k+1\}$ are labeled by words in $S(R)$, which are moreover in $S^{\lambda}(R)$ if $i \neq 0, k+1$. Therefore the connected 
component of the intersection $\tau \cap \mathfrak{p}$ cannot contain a pair $x_{i}, y_{i}$ with $i \in\{1, \ldots, k\}$. It follows that it can intersect at most two consecutive sub-arcs with endpoints $x_{i}, y_{i}$ with $i \in\{0,1, \ldots, k+1\}$, hence it is of length $<2 \lambda|\tau|$. We thus obtain that $\frac{1}{2}-3 \lambda<2 \lambda$, whence $\lambda>\frac{1}{10}$, a contradiction.

The following results are not used in an essential manner in our arguments, but they complete nicely the description of geodesics in small cancellation groups.

Lemma 3.31. Let $\mathfrak{g}$ be an $\eta$-relator-tied geodesic and let $\mathfrak{p}$ be a sub-geodesic in it. Then $\mathfrak{p}$ is either an $\eta$-compulsory geodesic, or it is the concatenation of three subgeodesics $\mathfrak{p}=\mathfrak{p}_{c} \sqcup \mathfrak{p}_{0} \sqcup \mathfrak{p}_{c}^{\prime}$, where $\mathfrak{p}_{c}, \mathfrak{p}_{c}^{\prime}$ are $\eta$-compulsory and contained in a contour (possibly either one of them or both trivial) and $\mathfrak{p}_{0}$ is an $\eta$-relator-tied component of $\mathfrak{p}$.

Proof. Let $a, b$ be the endpoints of $\mathfrak{g}$. With the previous convention $\mathfrak{g}=[a, b]$.

Step 1. Let us first assume that $\mathfrak{p}=[a, \sigma]$, with $a, \sigma, b$ a geodesic sequence.

Let $x_{0}=a, x_{1}, y_{0}, x_{2}, y_{1}, \ldots, x_{k+1}, y_{k}, y_{k+1}=b$ be the unique sequence of points on $\mathfrak{g}$ defined by Lemma 3.6.

Assume that $\sigma$ is in between a pair $y_{j}, x_{j+2}$. If the word labeling the geodesic $\left[x_{j+1}, \sigma\right]$ is contained in $S^{\eta}(R)$ then $\mathfrak{p}$ is $\eta$-relator-tied.

If the word labeling $\left[x_{j+1}, \sigma\right]$ is not in $S^{\eta}(R)$ (while it is still a sub-word of the relator labeling the contour $\left.t_{j+1}\right)$ then the pair $x_{j+1}, \sigma$ is $\eta$-compulsory. This implies that the required decomposition is $\mathfrak{p}=\left[a, y_{j}\right] \sqcup\left[y_{j}, \sigma\right]$.

Assume now that $\sigma$ is in between a pair $x_{j+1}, y_{j}$. If $\left[x_{j}, \sigma\right]$ is labeled by a word in $S^{\eta}(R)$ then $\mathfrak{p}$ is $\eta$-relator-tied; while in the opposite case the geodesic $\left[x_{j}, \sigma\right]$ is $\eta$-compulsory, and the conclusion holds with the decomposition $\mathfrak{p}=\left[a, y_{j-1}\right] \sqcup$ $\left[y_{j-1}, \sigma\right]$.

Step 2. Assume now that $\mathfrak{p}=[\varrho, \sigma]$, where $a, \varrho, \sigma, b$ is a geodesic sequence. According to Step $1,[a, \sigma]=[a, \mu] \sqcup[\mu, \sigma]$, where $[a, \mu]$ is an $\eta$-relator-tied component and $[\mu, \sigma]$ is $\eta$-compulsory (possibly trivial) and contained in a contour. If $\varrho \in[\mu, \sigma]$ then $\mathfrak{p}$ is $\eta$-compulsory. If $\varrho \in[a, \mu]$ then by reversing the order on $[a, \mu]$ and applying Step 1 we obtain that $[\varrho, \mu]=[\varrho, \nu] \sqcup[\nu, \mu]$, where $[\varrho, \nu]$ is $\eta$-compulsory (possibly trivial) and contained in a contour, and $[\nu, \mu]$ is an $\eta$-relator-tied component. It follows that

$$
\mathfrak{p}=[\varrho, \mu] \sqcup[\mu, \sigma]=[\varrho, \nu] \sqcup[\nu, \mu] \sqcup[\mu, \sigma]
$$

is the required decomposition.

Lemma 3.32. For each pair $\backslash y_{j}, z_{j} /$ in an $\eta$-criss-cross decomposition there exists a geodesic sequence

$$
p_{1}^{\prime}=y_{j}, p_{2}^{\prime}, q_{1}^{\prime}, p_{3}^{\prime}, q_{2}^{\prime}, \ldots, p_{n}^{\prime}, q_{n-1}^{\prime}, q_{n}^{\prime}=z_{j}, \text { for some } n=n(j),
$$

such that:

- $\left(p_{s}^{\prime}, q_{s}^{\prime}\right)$ are maximal with respect to the partial order relation $\Subset ;$

- $p_{\ell+1}^{\prime}, q_{\ell}^{\prime}$ bound $\eta$-relator-tied sub-geodesics both in the $\eta$-relator-tied geodesic joining $p_{\ell}^{\prime}, q_{\ell}^{\prime}$ and in the $\eta$-relator-tied geodesic joining $p_{\ell+1}^{\prime}, q_{\ell+1}^{\prime}$.

Proof. By definition, $\left[y_{j}, z_{j}\right]=\bigcup_{i \in I_{j}}\left[p_{i}, q_{i}\right]$. Without loss of generality we assume that there are no nested pairs among the $\left(p_{i}, q_{i}\right)$ with $i \in I_{j}$, in other words each pair $\left(p_{i}, q_{i}\right)$ is maximal with respect to the partial order relation $\Subset$. Proceeding as in the proof of Lemma 3.6 we also assume that, after selecting a subset in $I_{j}$, every

Copyright (c) 2018 Canadian Mathematical Society. All rights reserved. 
point on a (every) geodesic joining $y_{j}, z_{j}$ is between at most two pairs $\left(p_{i}, q_{i}\right)$. It then follows that the set of pairs indexed by $I_{j}$ compose a geodesic sequence as in (6). We set $p_{i}^{\prime}:=p_{i}$ and $q_{i}^{\prime}:=q_{i}$.

Consider now two consequent pairs that overlap: two pairs $\left(p_{i}^{\prime}, q_{i}^{\prime}\right)$ and $\left(p_{i+1}^{\prime}, q_{i+1}^{\prime}\right)$ such that $p_{i}^{\prime}, p_{i+1}^{\prime}, q_{i}^{\prime}, q_{i+1}^{\prime}$ is a geodesic sequence.

By definition, there exists a geodesic $\mathfrak{g}$ joining $a, b$ such that $p_{i}^{\prime}, q_{i}^{\prime}$ are the endpoints on it of an $\eta$-relator-tied component. Given two points $x, y \in \mathfrak{g}$, we denote by $[x, y]$ the sub-geodesic of $\mathfrak{g}$ with endpoints $x, y$.

We likewise know that there exists a geodesic $\mathfrak{p}$ such that $p_{i+1}^{\prime}, q_{i+1}^{\prime}$ bound an $\eta$-relator-tied component on $\mathfrak{p}$. According to the above, $\mathfrak{p}$ must contain $q_{i}^{\prime}$. In what follows, for $x, y$ in $\mathfrak{p}$ we denote by $\overline{x, y}$ the sub-arc of $\mathfrak{p}$ with endpoints $x, y$.

We have that $p_{i+1}^{\prime} \in\left[p_{i}^{\prime}, q_{i}^{\prime}\right]$. Lemma 3.31 implies that either $\left[p_{i+1}^{\prime}, q_{i}^{\prime}\right]$ is an $\eta$-compulsory component contained in a contour, or $\left[p_{i+1}^{\prime}, q_{i}^{\prime}\right]=\left[p_{i+1}^{\prime}, x\right] \sqcup\left[x, q_{i}^{\prime}\right]$, where $\left[p_{i+1}^{\prime}, x\right]$ is an $\eta$-compulsory component contained in a contour (possibly trivial) and $\left[x, q_{i}^{\prime}\right]$ is an $\eta$-relator-tied component.

Assume that $\left[p_{i+1}^{\prime}, q_{i}^{\prime}\right]$ is an $\eta$-compulsory component contained in a contour. Then the geodesic $\mathfrak{p}$ must also contain $\left[p_{i+1}^{\prime}, q_{i}^{\prime}\right] \subset \mathfrak{g}$. By replacing on $\mathfrak{p}$ the sub-arc with endpoints $a, p_{i+1}^{\prime}$ by $\left[a, p_{i+1}^{\prime}\right] \subset \mathfrak{g}$ we obtain a new geodesic $\mathfrak{r}$ joining $a, b$ such that $p_{i}^{\prime}$ and $q_{i+1}^{\prime}$ are the endpoints of an $\eta$-relator-tied sub-geodesic. It follows that $\left(p_{i}^{\prime}, q_{i+1}^{\prime}\right) \Subset(\alpha, \beta)$, where $\alpha, \beta$ are the endpoints on $\mathfrak{r}$ of an $\eta$-relator-tied component. In particular $(\alpha, \beta)=\left(p_{\ell}, q_{\ell}\right)$ for some $\ell \in I_{j}$, and $\left(p_{i}^{\prime}, q_{i}^{\prime}\right) \Subset\left(p_{\ell}, q_{\ell}\right)$. This contradicts the fact that we have considered pairs maximal with respect to $\Subset$.

Assume that $\left[p_{i+1}^{\prime}, q_{i}^{\prime}\right]=\left[p_{i+1}^{\prime}, x\right] \sqcup\left[x, q_{i}^{\prime}\right]$, where $\left[p_{i+1}^{\prime}, x\right]$ is an $\eta$-compulsory component contained in a contour (possibly trivial) and $\left[x, q_{i}^{\prime}\right]$ is an $\eta$-relatortied component. Since $q_{i}^{\prime} \in \mathfrak{p}$ and $\left[x, q_{i}^{\prime}\right]$ is an $\eta$-relator-tied component between $p_{i+1}^{\prime}$ and $q_{i}^{\prime}$ it follows that $x \in \mathfrak{p}$, hence $\left[p_{i+1}^{\prime}, x\right] \subset \mathfrak{p}$. There exists $y \in \mathfrak{p}$ such that $\overline{p_{i+1}^{\prime}, y}$ is labeled by a word in $S^{\eta}(R)$ and it is contained in a contour $t$. If $y \in \overline{p_{i+1}^{\prime}, x}=\left[p_{i+1}^{\prime}, x\right]$ then the contour $t$ intersects a distinct contour in a sub-arc of length $\geqslant \eta|t|$, a contradiction. Hence we must have that $x \in \overline{p_{i+1}^{\prime}, y}$.

According to the small cancellation condition $\overline{x, y}$ has length $>\left(1-\frac{\lambda}{\eta}\right)$ of the length of $\overline{p_{i+1}^{\prime}, y}$, so at least $\eta\left(1-\frac{\lambda}{\eta}\right)|t|$. This implies that if $\eta\left(1-\frac{\lambda}{\eta}\right) \geqslant \lambda$, equivalently $\eta \geqslant 2 \lambda$, then by Lemma 3.10, $t$ must be the first contour for the pair $x, q_{i}^{\prime}$. But this implies that $p_{i+1}^{\prime}=x$.

Similarly, we argue that $\overline{p_{i+1}^{\prime}, q_{i}^{\prime}}$ is an $\eta$-relator-tied geodesic.

\section{QUASI-HOMOMORPHISMS ON SMALL CANCELLATION GROUPS}

Recall that a quasi-homomorphism (also called a quasi-morphism or a pseudocharacter) on a group $G$ is a function $\mathfrak{h}: G \rightarrow \mathbb{R}$ such that its defect

$$
\mathfrak{d}(\mathfrak{h}):=\sup _{a, b \in G}|\mathfrak{h}(a b)-\mathfrak{h}(a)-\mathfrak{h}(b)|
$$

is finite. The real vector space $\mathcal{Q}(G)$ of all quasi-homomorphisms of $G$ has three important subspaces: the subspace $\ell^{\infty}(G)$ of bounded real functions on $G$, the subspace $\operatorname{Hom}(\mathrm{G}, \mathbb{R})=\mathrm{H}^{1}(\mathrm{G}, \mathbb{R})$ of homomorphisms on $G$, and the subspace $\ell^{\infty}(G)+$ $\operatorname{Hom}(G, \mathbb{R})$ of functions that differ from a homomorphism by a bounded function. Consider the quotient spaces

$$
Q H(G)=\mathcal{Q}(G) / \mathcal{B}(G) \text { and } \widetilde{Q H}(G)=\mathcal{Q}(G) /\left[\ell^{\infty}(G)+\operatorname{Hom}(\mathrm{G}, \mathbb{R})\right] .
$$

Copyright (c) 2018 Canadian Mathematical Society. All rights reserved. 
The space $\widetilde{Q H}(G)$ can be identified with the kernel of the comparison map

$$
H_{b}^{2}(G) \rightarrow H^{2}(G),
$$

where $H_{b}^{2}(G)$ is the second bounded cohomology of $G$.

In this paper, as an application of our results on the geometry of small cancellation groups with the $C^{\prime}(1 / 12)$-condition, we show that for such a group $G$ the space $\widetilde{Q H}(G)$ is infinite dimensional, with a basis of power continuum.

Following the work of Epstein and Fujiwara [EF97, Fuj00, Fuj98] as well as of Bestvina and Fujiwara [BF02], we shall prove the following.

Proposition 4.1. Let $G$ be a finitely generated infinitely presented group and let $\langle S \mid R\rangle$ be a presentation such that $R$ satisfies the $C^{\prime}(1 / 12)$-condition. For a given $\eta \in\left[3 \lambda, \frac{1}{2}-2 \lambda\right]$ appropriately chosen, there exists a sequence $\mathfrak{u}_{n}$ of elements in $G$ and a sequence $\mathfrak{h}_{\mathfrak{u}_{n}}: G \rightarrow \mathbb{R}$ of quasi-morphisms, with $n \in \mathbb{N}, n \geqslant 1$, such that

(1) the set of word lengths $\left|\mathfrak{u}_{n}\right|$ diverges to $\infty$;

(2) every group homomorphism $\phi: G \rightarrow \mathbb{R}$ has the property that $\phi\left(\mathfrak{u}_{n}\right)=0$ for every $n \in \mathbb{N}, n \geqslant 1$

(3) the sequence of defects $\mathfrak{d}\left(\mathfrak{h}_{\mathfrak{u}_{n}}\right)$ is bounded;

(4) for every $n$ and every $k \in \mathbb{N}, k \geqslant 1, \mathfrak{h}_{\mathfrak{u}_{n}}\left(\mathfrak{u}_{n}^{k}\right)=k$;

(5) for every $n \neq m$, and every $k \in \mathbb{N}, k \geqslant 1, \mathfrak{h}_{\mathfrak{u}_{n}}\left(\mathfrak{u}_{m}^{k}\right)=0$.

Proof. We enumerate the relators $\left\{r_{1}, r_{2}, \ldots\right\}$ in $R$ so that their lengths compose a non-decreasing sequence. Consider the sequence of finite subsets of $\mathbb{N}$ defined by

$$
I_{n}=[1+2+\ldots+n, 1+2+\ldots+n+1) \cap \mathbb{N} .
$$

Define two sequences of finite subsets $A_{n}$ and $B_{n}$ of $R$, described by $A_{n}=$ $\left\{r_{2 i-1} \mid i \in I_{n}\right\}$ and $B_{n}=\left\{r_{2 i} \mid i \in I_{n}\right\}$.

To simplify the notation, in what follows we denote the relator $r_{2 i-1}$ by $\alpha_{i}$ and $r_{2 i}$ by $\beta_{i}$, respectively. Thus, $A_{n}=\left\{\alpha_{i} \mid i \in I_{n}\right\}$ and $B_{n}=\left\{\beta_{i} \mid i \in I_{n}\right\}$.

Let $X$ be a finite set of relators equal either to a set $A_{n}$ or to a set $B_{n}$. We construct an element $x \in G$ corresponding to $X$, as follows. Assume $X$ is composed of the relators $\rho_{1}, \ldots, \rho_{k}$ enumerated in increasing order. For every $i \in\{1,2, \ldots, k\}$ let $y_{i}$ be the prefix of $\rho_{i}$ of length $\left\lfloor\frac{\left|\rho_{i}\right|}{2}\right\rfloor$. Define the element $x=y_{1} y_{2} \cdots y_{k}$. An argument very similar to the one in Lemma 3.30 implies that $x$ is an $\eta$-relator-tied element and that every geodesic joining 1 and $x$ is contained in the $\eta$-succession of contours $t_{1}, y_{1} t_{2}, y_{1} y_{2} t_{3}, \ldots,\left[y_{1} \cdots y_{k-1}\right] t_{k}$, where $t_{i}$ is the loop through 1 in the Cayley graph, labeled by $\rho_{i}$.

When $X=A_{n}$, respectively $X=B_{n}$ the corresponding element $x$ is denoted by $a_{n}$, respectively by $b_{n}$.

We define $\mathfrak{u}_{n}=\left[a_{n}, b_{n}\right]$. This implies property (2) in Proposition 4.1.

Lemma 3.30 applied to geodesics joining 1 to $\mathfrak{u}_{n}$ implies that the length $\left|\mathfrak{u}_{n}\right|$ is at least the double of $\left(\frac{1}{2}-\lambda\right) \sum_{i \in I_{n}}\left[\left|\alpha_{i}\right|+\left|\beta_{i}\right|\right]$. It follows that property (1) in Proposition 4.1 is also satisfied.

We now define the sequence of quasi-morphisms. We start with a general construction. Let $\mathfrak{v}$ be an $\eta$-relator-tied element in $G$.

Definition 4.2. (1) Let $(a, b) \in G \times G$. A quasi-copy of $\mathfrak{v}$ nested inside $(a, b)$ is a pair of points $x, y \in \mathcal{G}^{\eta}(a, b)$ such that $y=x \mathfrak{v}$ and such that there exists an $\eta$-succession of contours $t_{1}, \ldots, t_{k}$ contained in $\mathcal{G}^{\eta}(a, b)$ such that:

Copyright (c) 2018 Canadian Mathematical Society. All rights reserved. 
- $x$ is either one of the endpoints of the intersection of $t_{1}$ with a contour $t_{0}$ such that $t_{0}, t_{1}, \ldots, t_{k}$ is an $\eta$-succession contained in $\mathcal{G}^{\eta}(a, b)$, or the intersection of $t_{1}$ with a compulsory geodesic preceding $t_{1}, \ldots, t_{k}$ in $\mathcal{G}^{\eta}(a, b)$;

- $y$ is either one of the endpoints of the intersection of $t_{k}$ with a contour $t_{k+1}$ such that $t_{1}, \ldots, t_{k}, t_{k+1}$ is an $\eta$-succession contained in $\mathcal{G}^{\eta}(a, b)$, or the intersection of $t_{k}$ with a compulsory geodesic succeeding $t_{1}, \ldots, t_{k}$ in $\mathcal{G}^{\eta}(a, b)$.

(2) We say that two quasi-copies of $\mathfrak{v}$ nested inside $a, b$ are non-overlapping if the corresponding $\eta$-successions of contours $t_{1}, \ldots, t_{k}$ respectively $\tau_{1}, \ldots, \tau_{k}$ are disjoint, as finite sets of contours.

(3) When $(a, b)=(1, g)$ for some element $g \in G$ we speak about quasi-copies of $\mathfrak{v}$ nested inside $g$.

Note that according to the definition of $\mathcal{G}^{\eta}(a, b)$ and to Lemma 3.10, the pair of points $x, y$ uniquely determines the $\eta$-succession $t_{1}, \ldots, t_{k}$.

Lemma 4.3. Let $x, y$ be a pair of points in $\mathcal{G}^{\eta}(g)$ (with the Notation 3.28) composing a nested quasi-copy of $\mathfrak{v}$ in $g$, and let $t_{1}, \ldots, t_{k}$ be the corresponding $\eta$-succession of contours. There exists no other pair of points $p, q$ in $\bigcup_{i=1}^{k} t_{i}$ such that $q=p \mathfrak{v}$.

Proof. Lemma 3.30 can be easily generalized to pairs of points $a, b$ contained in an $\eta$-succession of contours. Applied to the pair $x, y$, it implies that every geodesic joining $x, y$ is $\eta$-relator-tied. This implies that $\mathfrak{v}$ is an $\eta$-relator-tied element. Let $\mathfrak{g}$ be an $\eta$-relator-tied geodesic joining 1 and $\mathfrak{v}$. It follows that $x \mathfrak{g}$ is contained in $\bigcup_{i=1}^{k} t_{i}$, whence the unique sequence of vertices on $\mathfrak{g}$ described in Lemma 3.6 contains $k$ pairs $x_{i}, y_{i}$.

Assume that there exists another pair of points $p \in t_{r}$ and $q \in t_{s}$ with $1 \leqslant$ $r \leqslant s \leqslant k$ such that $p, q$ compose a nested quasi-copy of $\mathfrak{v}$ in $g$. The $p \mathfrak{g}$ is a geodesic joining $p$ and $q$, which according to Lemma 3.30 is contained in $\bigcup_{i=r}^{s} t_{i}$. The uniqueness of the sequence in Lemma 3.6 implies that $s-r+1=k$, whence $r=1$ and $s=k$. The same uniqueness implies that each pair $p x_{i}, p y_{i}$, translate of the corresponding pair on $\mathfrak{g}$, is the pair of endpoints of the intersection $p \mathfrak{g} \cap t_{i}$.

The first pair in the unique sequence of vertices on $\mathfrak{g}$ as in Lemma 3.6 is of the form $1, h$, where $h$ is represented by a word $w_{1}$ in $S^{\frac{1}{2}-2 \lambda}(R)$, prefix of a relator $\rho$ labeling a unique loop $\tau$ through 1 in the Cayley graph. By the above $x \tau=p \tau=t_{1}$, therefore $p^{-1} x \tau=\tau$. This and the small cancellation condition $C^{\prime}(1 / 12)$ imply that the element $p^{-1} x$ is trivial in $G$. Indeed, the condition $C^{\prime}(1 / 12)$ implies that the stabilizer in $G$ of any contour is trivial, otherwise one could find two distinct copies of the same long sub-word in the label of that contour.

We conclude that $p=x$, and $q=p \mathfrak{v}=x \mathfrak{v}=y$.

Definition 4.4. The point $x$ is called the initial point of the nested quasi-copy, while $y$ is called the terminal point of the nested quasi-copy.

We define $c_{\mathfrak{v}}: G \times G \rightarrow \mathbb{R}$ such that $c_{\mathfrak{v}}(a, b)$ is the maximal number of pairwise non-overlapping quasi-copies of $\mathfrak{v}$ nested inside $(a, b)$.

By abuse of notation, we define $c_{\mathfrak{v}}: G \rightarrow \mathbb{R}$ such that $c_{\mathfrak{v}}(g)$ is the maximal number of pairwise non-overlapping quasi-copies of $\mathfrak{v}$ nested inside $g$.

Clearly $c_{\mathfrak{v}}(a, b)=c_{\mathfrak{v}}(h a, h b)$ and $c_{\mathfrak{v}}(g)=c_{\mathfrak{v}}(h, h g)$, for every $h \in G$. 
Proposition 4.5. Let $\mathfrak{v}$ be one of the elements $\mathfrak{u}_{n}$ for $n \in \mathbb{N}$. The map $\mathfrak{h}_{\mathfrak{v}}: G \rightarrow \mathbb{R}$, $\mathfrak{h}_{\mathfrak{v}}=c_{\mathfrak{v}}-c_{\mathfrak{v}}-1$ is a quasi-morphism with defect at most 2 .

Proof. Let $g$ and $h$ be two arbitrary elements in $G$. Our goal is to show that

$$
\left|\mathfrak{h}_{\mathfrak{v}}(g h)-\mathfrak{h}_{\mathfrak{v}}(g)-\mathfrak{h}_{\mathfrak{v}}(h)\right| \leqslant 2 .
$$

The study of geodesic triangles that was done in the preceding section implies that the intersection $\mathcal{G}^{\eta}(g) \cap \mathcal{G}^{\eta}(h) \cap \mathcal{G}^{\eta}(g, g h)$ is either a contour, or a tripod (with some branches possibly reduced to a point) appearing as intersection of three contours, or a sub-path in a contour $\omega$ composed of three consecutive sub-paths (possibly reduced to a point) of lengths $<\lambda|\omega|$, for the first and third, and $<\eta|\omega|$ for the second. Note that whatever the geometric nature of the intersection, it splits each of the three sets $\mathcal{G}^{\eta}(g), \mathcal{G}^{\eta}(h), \mathcal{G}^{\eta}(g, g h)$, into two connected components.

We call the intersection $\mathcal{G}^{\eta}(g) \cap \mathcal{G}^{\eta}(h) \cap \mathcal{G}^{\eta}(g, g h)$ the median object for the triple $g, h, g h$, and we denote it $\mathfrak{m}(g, h)$.

We say that $\mathfrak{m}(g, h)$ separates a quasi-copy of $\mathfrak{v}$ nested inside $(a, b)$, where $(a, b) \in$ $\{(1, g),(1, g h),(g, g h)\}$ if the two points $x, y$ determining that quasi-copy are in two different connected components of $\mathcal{G}^{\eta}(a, b) \backslash \mathfrak{m}(g, h)$.

Assume that the maxima $c_{\mathfrak{v} \pm 1}(g), c_{\mathfrak{v} \pm 1}(g h)$ and $c_{\mathfrak{v} \pm 1}(g, g h)$ are all attained only by considering nested quasi-copies that are not separated by $\mathfrak{m}(g, h)$. In that case one can easily see that $\mathfrak{h}_{\mathfrak{v}}(g h)-\mathfrak{h}_{\mathfrak{v}}(g)-\mathfrak{h}_{\mathfrak{v}}(h)=0$.

Assume now that every counting that realizes the maximum $c_{\mathfrak{v}}(g h)$ must take into account a pair $x, y$ separated by $\mathfrak{m}(g, h)$. Inside $\mathcal{G}^{\eta}(g h)$ one has then an $\eta^{-}$ succession of contours $t_{1}, \ldots, t_{k}$ with $x \in t_{1}$ and $y \in t_{k}$. The choice of the labels of contours in $\mathcal{G}^{\eta}\left(\mathfrak{u}_{n}\right)$ implies that:

- no quasi-copy of $\mathfrak{v}^{-1}$ nested inside $g h$ can contain a sub-sequence in the sequence of contours $t_{1}, \ldots, t_{k}$

- no initial point of a quasi-copy of $\mathfrak{v}$ nested inside $g$ can be contained in $\bigcup_{i=1}^{k} t_{i} \cap \mathcal{G}^{\eta}(g)$

- no terminal point of a quasi-copy of $\mathfrak{v}$ nested inside $(g, g h)$ can be contained in $\bigcup_{i=1}^{k} t_{i} \cap \mathcal{G}^{\eta}(g, g h)$.

It is nevertheless possible that $\bigcup_{i=1}^{k} t_{i} \cap \mathcal{G}^{\eta}(g)$ contains an initial point of a quasicopy of $\mathfrak{v}^{-1}$ nested inside $g$. But in that case no terminal point of a quasi-copy of $\mathfrak{v}^{-1}$ nested inside $(g, g h)$ can be contained in $\bigcup_{i=1}^{k} t_{i} \cap \mathcal{G}^{\eta}(g, g h)$. We thus obtain that

$$
\mathfrak{h}_{\mathfrak{v}}(g h)-\mathfrak{h}_{\mathfrak{v}}(g)-\mathfrak{h}_{\mathfrak{v}}(h)=2 .
$$

Similarly, $\bigcup_{i=1}^{k} t_{i} \cap \mathcal{G}^{\eta}(g, g h)$ may contain a terminal point of a quasi-copy of $\mathfrak{v}^{-1}$ nested inside $(g, g h)$; in which case $\bigcup_{i=1}^{k} t_{i} \cap \mathcal{G}^{\eta}(g)$ cannot contain an initial point of a quasi-copy of $\mathfrak{v}^{-1}$ nested inside $g$, and (7) is still verified.

If none of the above two cases occurs then the right-hand side in (7) is 1.

In the case when every counting that realizes the maximum $c_{\mathfrak{v}^{-1}}(g h)$ must take into account a pair $x, y$ separated by $\mathfrak{m}(g, h)$ similar arguments work and give equalities like in (7), with the right hand side either -2 or -1 .

The cases when $c_{\mathfrak{v} \pm 1}(g h)$ is replaced by either $c_{\mathfrak{v} \pm 1}(g)$ or $c_{\mathfrak{v} \pm 1}(g, g h)$ are treated similarly and give equalities like in (7), with the right hand side \pm 2 or \pm 1 .

We now finish the proof of Proposition 4.1. Proposition 4.5 implies that all the quasi-homomorphisms $\mathfrak{h}_{\mathfrak{u}_{n}}$ have a defect bounded by 2. Properties (4) and (5)

Copyright (c) 2018 Canadian Mathematical Society. All rights reserved. 
follow from Corollary 3.9 and from the construction of the $\eta$-relator-tied elements $\mathfrak{u}_{n}$.

The end of the proof now follows the standard argument in the work of EpsteinFujiwara [EF97, Fuj00, Fuj98] and Bestvina-Fujiwara [BF02]. We repeat it here for the sake of completeness.

Theorem 4.6. Let $G$ be an infinitely presented finitely generated group given by a presentation satisfying the small cancellation condition $C^{\prime}(1 / 12)$. Then there exists an injective linear map $\ell^{1} \rightarrow \widetilde{Q H}(G)$. In particular, the dimension of $\widetilde{Q H}(G)$ is power continuum.

Proof. We consider the map $\ell^{1} \rightarrow \mathcal{Q}(G)$ defined by $\left(a_{n}\right) \mapsto \sum_{n} a_{n} \mathfrak{h}_{\mathfrak{u}_{n}}$. Proposition 4.1, (3), implies that each image is indeed a quasi-morphism. Proposition 4.1, (1), implies that when $a_{n} \mathfrak{h}_{\mathfrak{u}_{n}}$ is evaluated in some element $g \in G$, only finitely many terms take non-zero value, thus the sum is always finite.

The above map defines a linear map $\ell^{1} \rightarrow \widetilde{Q H}(G)$. We now prove that it is injective. Let $\left(a_{n}\right) \in \ell^{1}$ be such that $\mathfrak{h}=\sum_{n} a_{n} \mathfrak{h}_{\mathfrak{u}_{n}}$ is at bounded distance from a homomorphism. In particular, it follows by Proposition 4.1, (2), that for every $n$ and $k, \mathfrak{h}\left(\mathfrak{u}_{n}^{k}\right)$ is uniformly bounded.

On the other hand, given $n \in \mathbb{N}$ such that $a_{n} \neq 0$, Proposition 4.1, (4) and (5), imply that $\mathfrak{h}\left(\mathfrak{u}_{n}^{k}\right)=a_{n} k$. This contradicts the fact that $\mathfrak{h}\left(\mathfrak{u}_{n}^{k}\right)$ is bounded uniformly in $k$.

\section{REFERENCES}

[AD17] G. Arzhantseva and C. Druţu, Rapid Decay property of graphical small cancellation groups, preprint, 2017.

[Bav91] C. Bavard, Longueur stable des commutateurs, Enseign. Math. (2) 37 (1991), no. 1-2, 109-150.

[BF02] M. Bestvina and K. Fujiwara, Bounded cohomology of subgroups of mapping class groups, Geom. Topol. 6 (2002), 69-89 (electronic).

[BM99] M. Burger and N. Monod, Bounded cohomology of lattices in higher rank Lie groups, J. Eur. Math. Soc. (JEMS) 1 (1999), no. 2, 199-235.

[BM02] - Continuous bounded cohomology and applications to rigidity theory, Geom. Funct. Anal. 12 (2002), no. 2, 219-280.

[Bro81] R. Brooks, Some remarks on bounded cohomology, Riemann surfaces and related topics: Proceedings of the 1978 Stony Brook Conference (State Univ. New York, Stony Brook, N.Y., 1978), Ann. of Math. Stud., vol. 97, Princeton Univ. Press, Princeton, N.J., 1981, pp. 53-63.

[DGO17] F. Dahmani, V. Guirardel, and D. Osin, Hyperbolically embedded subgroups and rotating families in groups acting on hyperbolic spaces, Mem. Amer. Math. Soc. 245 (2017), no. $1156, \mathrm{v}+152$.

[EF97] D. B. A. Epstein and K. Fujiwara, The second bounded cohomology of word-hyperbolic groups, Topology 36 (1997), no. 6, 1275-1289.

[Fuj98] K. Fujiwara, The second bounded cohomology of a group acting on a Gromov-hyperbolic space, Proc. London Math. Soc. (3) 76 (1998), no. 1, 70-94.

[Fuj00] , The second bounded cohomology of an amalgamated free product of groups, Trans. Amer. Math. Soc. 352 (2000), no. 3, 1113-1129.

[Fuj09] Q Quasi-homomorphisms on mapping class groups, Handbook of Teichmüller theory. Vol. II, IRMA Lect. Math. Theor. Phys., vol. 13, Eur. Math. Soc., Zürich, 2009, pp. 241-269.

[GdlH91] E. Ghys and P. de la Harpe, Infinite groups as geometric objects (after Gromov), Ergodic theory, symbolic dynamics and hyperbolic spaces (Trieste, 1989) (T. Bedford, M. Keane, and C. Series, eds.), Oxford Univ. Press, 1991, pp. 299-314.

Copyright (c) 2018 Canadian Mathematical Society. All rights reserved. 
Author's Draft: To be published in the Canadian Journal of Mathematics http://dx.doi.org/10.4153/CJM-2018-036-7

GEOMETRY OF SMALL CANCELLATION GROUPS AND QUASI-HOMOMORPHISMS 21

[Gro82] M. Gromov, Volume and bounded cohomology, Publ. Math. IHES (1982), no. 56, 5-99.

[Gro87] _ Hyperbolic groups, Essays in group theory (S. Gersten, ed.), MSRI Publications, vol. 8, Springer, 1987.

[GS14] D. Gruber and A. Sisto, Infinitely presented graphical small cancellation groups are acylindrically hyperbolic, arXiv:1408.4488; to appear in Ann. Inst. Fourier. (2014).

[HO13] M. Hull and D. Osin, Induced quasicocycles on groups with hyperbolically embedded subgroups, Algebr. Geom. Topol. 13 (2013), no. 5, 2635-2665.

[LS77] R. Lyndon and P. Schupp, Combinatorial group theory, Springer-Verlag, Berlin, 1977.

[RS95] E. Rips and Z. Sela, Canonical representatives and equations in hyperbolic groups, Invent. Math. 120 (1995), no. 3, 489-512.

[Sel92] Z. Sela, Uniform embeddings of hyperbolic groups in Hilbert spaces, Israel J. Math. 80 (1992), no. 1-2, 171-181.

University of Vienna, Faculty of Mathematics, Oskar-Morgenstern-Platz 1, 1090 Vienna, Austria

E-mail address: goulnara.arzhantseva@univie.ac.at

Mathematical Institute, AWB, Radcliffe Observatory Quarter, Woodstock Road, OXFord OX2 6GG, United Kingdom.

E-mail address: drutu@maths.ox.ac.uk

Copyright (c) 2018 Canadian Mathematical Society. All rights reserved. 